\title{
Impacto de intervenciones educativas en el uso racional del medicamento por farmacéuticos comunitarios en estudiantes de bachiller
}

\author{
Joaquina Huarte Royo ${ }^{1,2}$, Francesc Moranta Ribas ${ }^{1,3}$ \\ 1. Comisión de Educación para la Salud de la Sociedad Española de Farmacia Clínica, Familiar y Comunitaria (SEFAC). 2. Farmacéutica comunitaria en \\ Pamplona (Navarra). 3. Farmacéutico comunitario en Palma (Illes Balears).
}

\section{PALABRAS CLAVE}

Uso racional del medicamento, farmacéuticos comunitarios, actividades educativas

\section{ABREVIATURAS}

AEMPS: Agencia Española de Medicamentos y Productos Sanitarios BMQ: Beliefs about Medicines Questionnaire CEIC: Comité Ético de Investigación Clínica GC: Grupo de control GI: Grupo de intervención PRM: Problemas relacionados con los medicamentos RNM: Resultados negativos asociados a la medicación SEFAC: Sociedad Española de Farmacia Clínica, Familiar y Comunitaria

\section{KEYWORDS}

Rational drug use, community pharmacists, educational activities

\section{RESUMEN}

Introducción: el desconocimiento general del uso adecuado de medicamentos es un problema de educación, que debe ser abordado desde una temprana edad, como parte del proceso educativo de nuestros jóvenes, ya que será crucial inculcar buenos hábitos en autocuidado y adherencia farmacoterapéutica desde la adolescencia para conseguir un uso responsable del medicamento a medio y largo plazo. El objetivo de este estudio fue evaluar el impacto de intervenciones educativas grupales realizadas por farmacéuticos comunitarios en el conocimiento básico del uso del medicamento, y las creencias respecto a la sobremedicalización y a su peligrosidad

Material y métodos: estudio analítico, controlado, aleatorizado por conglomerados, centrado en una muestra de estudiantes de bachillerato de 17 provincias de España. Las intervenciones se aplicaron a los alumnos del grupo intervención. Para evaluar el impacto de las intervenciones se utilizaron dos cuestionarios que cumplimentaron los alumnos del grupo control e intervención en tres ocasiones; al iniciar (línea base de partida) y finalizar las intervenciones, y a los tres meses.

Resultados: participaron 15.711 alumnos. La interacción grupo-tiempo resultó $(p<0,05)$ en las tres variables, obteniendo un resultado medio de mejora con la intervención de 1.4 puntos en conocimientos, 0.8 sobremedicalizacion y 0.3 peligrosidad. La intervención mejoró el conocimiento y las creencias sobre medicamentos de los estudiantes independientemente del nivel del alumno en línea base.

Conclusión: las intervenciones educativas sobre el uso de medicamentos en estudiantes de bachillerato se han demostrado efectivas y viables para mejorar tanto el conocimiento general como sus creencias respecto a sobremedicalización y peligrosidad de los medicamentos.

\section{Effect of educational interventions on the rational use of medicines by} Community Pharmacists in High School students

\section{ABSTRACT}

Background: The general lack of knowledge about the suitable use of medicines is a problem related to education, which must be tackled from an early age, as part of the educational process of our young people. As a result, it will be crucial to instil good habits in self-care and treatment adherence from adolescence to achieve responsible use of the medicine in the middle and long term. The aim of this clinical study was to assess the impact of group educational interventions carried out by community pharmacists on the basic knowledge of the use of medicine, and beliefs regarding that medicines are harmful, addictive, and poisons, and that medicines are overused by doctors

Methods: Analytical, controlled, randomised cluster study focused on a sample of high school students from 17 provinces in Spain. The educational interventions were only delivered to students who belong to the intervention group. Two questionnaires were used to evaluate the impact of the interventions. The questionnaires were filled by the students of both intervention and control groups, on three occasions; at the beginning (baseline) and end of the interventions, and at three months.

Results: 15,711 students participated. The interaction between group and time resulted $(p<0.05)$ in the three variables, obtaining a mean result of improvement over the intervention of 1.4 points in knowledge, 0.8 in overuse, and 0.3 in medicine risks. The intervention improved the knowledge of the students, and beliefs about medication regardless of the initial baseline level of the students.

Conclusion: It has been proved that educational interventions on the use of medicines in high school students are effective and feasible in improving both general knowledge and beliefs about overuse and dangerousness of medicines.
Financiación: este trabajo está basado en el proyecto ConóceMe, que ha contado con la colaboración de Laboratorios Cinfa.

Conflicto de intereses: ninguno.

Cite este artículo como: Huarte J, Moranta F. Impacto de intervenciones educativas en el uso racional del medicamento por farmacéuticos comunitarios en estudiantes de bachiller. Farmacéuticos Comunitarios. 2021 Apr 19; 13 (2): 11-24. doi:10.33620/FC.2173-9218.(2021/Vol13).002.04

Correspondencia: Joaquina Huarte Royo (jhuarte@sefac.org).

ISSN 1885-8619 @SEFAC (Sociedad Española de Farmacia Clínica, Familiar y Comunitaria). Todos los derechos reservados. 


\section{Introducción}

En las últimas décadas se ha evidenciado por diferentes estudios que un elevado porcentaje de los medicamentos se prescriben o se dispensan erróneamente $(1,2)$, pero, además, más de la mitad son usados incorrectamente por los pacientes $(1,2)$; a ello hay que sumar los elevados índices de falta de adherencia terapéutica existentes en nuestro entorno (3). Por otro lado, España es uno de los países occidentalizados con un mayor consumo de medicamentos (4), por lo tanto, el elevado uso de ellos, añadido al elevado porcentaje de falta de adherencia terapéutica (3) y a su errónea utilización $(5,6)$ conlleva que el uso irracional del medicamento sea considerado como un problema de salud pública de considerables dimensiones (7). En esta línea, el incumplimiento terapéutico y el mal uso de los tratamientos, entre otros muchos factores, pueden relacionarse con una baja alfabetización en salud, que es la capacidad que tiene un individuo de obtener, procesar y entender información básica sobre su salud en general y sobre su tratamiento en particular (8).

La misión del farmacéutico comunitario, entre otras, consiste en atender las necesidades del paciente ante sus medicamentos (9). En el desarrollo de esa actividad asistencial en la farmacia comunitaria se detecta con bastante frecuencia el desconocimiento del paciente sobre las normas generales del uso del medicamento $(5,6,10)$. En muchas ocasiones también se evidencia que el paciente no considera importantes esas normas básicas $(5,6,10)$. Desconocer la manera idónea de utilizar una forma farmacéutica, administrarla erróneamente, conservarla en condiciones inadecuadas, no completar el periodo de tratamiento o bien su consumo inadecuado son aspectos que se clasifican como problemas relacionados con el uso del medicamento (PRM) que pueden afectar negativamente al paciente, así como interferir en las expectativas de buenos resultados del tratamiento instaurado. Se trata de PRM o elementos del proceso que suponen al usuario de medicamentos un mayor riesgo de sufrir un resultado negativo asociado a la medicación (RNM), bien de necesidad, inefectividad o inseguridad. Se trata básicamente de un problema de educación.
Además de una información clara, razonada y adaptada al paciente, la educación en la actividad asistencial requiere un enfoque humanístico con el paciente (11), entendido como una tarea complementaria de escucha, empatía, apoyo y motivación, desde el respeto a la autonomía del mismo. De este modo el empoderamiento real de los pacientes en temas concernientes a medicamentos podría reducir el porcentaje de errores relacionados con estos y contribuir en la mejora de la actitud y del comportamiento de los pacientes en su uso racional.

Llegados a este punto, había que dar un paso más y elaborar un proyecto dirigido a la población más joven. El desconocimiento general del uso adecuado de los medicamentos es un problema que debe ser abordado desde una temprana edad, como una parte del proceso educativo de nuestros jóvenes, adaptado a las herramientas educativas actuales, ya que será crucial inculcar buenos hábitos en el autocuidado y la adherencia terapéutica desde la adolescencia para conseguir un uso racional del medicamento a medio y largo plazo.

\section{Hipótesis de estudio}

¿Se podrían mejorar los conocimientos y actitudes sobre el uso racional del medicamento en adolescentes, mediante la puesta en marcha de intervenciones educativas grupales basadas en el correcto proceso de uso del medicamento ofrecidas por farmacéuticos comunitarios? Para responder a esta pregunta se ha diseñado este proyecto de investigación: proyecto ConóceMe: Conoce el medicamento, descubre al farmacéutico, con la intención de involucrar definitivamente al farmacéutico comunitario en la educación sobre el medicamento, más allá de su ámbito normalizado de actuación, como un facultativo experto del medicamento en intervenciones para la mejora de la salud pública.

\section{Material y métodos}

Diseño y emplazamiento

Estudio analítico, controlado, aleatorizado por conglomerados, centrado en una muestra de estudiantes de bachillerato de 17 provincias de España, una por cada comunidad autónoma.
Clasificado por la Agencia Española de Medicamentos y Productos Sanitarios (AEMPS) como Estudio Observacional No Posautorización (No-EPA) y aprobado por el Comité Ético de Investigación Clínica (CEIC) de la Fundació Sant Joan de Déu.

\section{Objetivos}

El objetivo principal fue el de evaluar el impacto de intervenciones educativas grupales realizadas por farmacéuticos comunitarios en el conocimiento básico del uso del medicamento, y las actitudes y creencias respecto a la sobremedicalización y a su peligrosidad.

Como objetivos secundarios se propuso evaluar el grado de satisfacción de los alumnos asistentes a las intervenciones educativas y conocer en qué medida son usuarios de medicamentos, así como evaluar el grado de satisfacción con la actividad educativa del personal docente de los centros educativos participantes en el proyecto.

\section{Población de estudio}

Los criterios de inclusión fueron cursar primero o segundo de bachillerato, pertenecer a un centro educativo participante en el ensayo, aceptar participar en el estudio y aportar el consentimiento informado firmado por un padre o tutor.

\section{Materiales}

El documento base de definición de conceptos y temáticas es la base documental sobre las que pivotaron las intervenciones educativas, y la base argumental que utilizaron los farmacéuticos voluntarios participantes en el estudio.

Para su definición y redactado se siguió el siguiente proceso:

Se partió de un panel de farmacéuticos expertos para dimensionar el concepto de conocimientos y actitudes necesarias para el uso racional del medicamento; es decir, definir y acotar las temáticas sobre las que se iba a impartir la intervención educativa al adolescente. ¿Qué es lo que necesita conocer el estudiante de bachillerato para mejorar a medio y largo plazo el uso racional del medicamento en nuestro ámbito? Una vez definidos y dimensionados los conceptos en cuatro áreas temáticas (A, B, C, D) y 15 puntos conceptuales se procedió, a partir del 
mismo panel de expertos, a dotarles de contenido, atendiendo a las circunstancias especiales del colectivo al que iba dirigido, adolescentes que cursan bachillerato, y adaptándolo a ese ámbito (tabla1).

En último lugar se procedió a la revisión del documento por expertos profesionales de la pedagogía con el fin de supervisar su redacción y de establecer las líneas básicas de las intervenciones educativas.

Posteriormente este documento fue la base para diseñar una presentación en soporte digital que se utilizó como apoyo y presentación a las intervenciones educativas. En ella se explican con ejemplos prácticos y metodologías modernas de pedagogía las normas generales de uso del medicamento, razonadas y adaptadas al alumno. Las intervenciones educativas se basaron en la explicación de los conceptos de forma clara, concisa y adecuada a las características sociales y culturales de los alumnos de bachillerato.

Por otra parte, se utilizó también una pequeña guía impresa de consejos y recomendaciones para el buen uso del medicamento editada por la Sociedad Española de Farmacia Clínica, Familiar y Comunitaria (SEFAC), la Guía práctica para el uso adecuado de los medicamentos, elaborada por Joaquina Huarte Royo y revisada por farmacéuticos expertos en educación sobre la salud. Se entregó justo después de las intervenciones educativas como material didáctico de refuerzo y consulta a las intervenciones realizadas.

Para medir las variables de resultado se utilizaron dos cuestionarios, uno de ellos diseñado y validado por SEFAC en una población de adolescentes españoles (pendiente de publicación) como un instrumento que evalúa el conocimiento básico sobre medicamentos con una escala del 1 al 10 y una valoración de 32 ítems, que refleja un mejor conocimiento a mayores puntuaciones (figura 1), y el "Beliefs about Medicines Questionnaire” (BMQ) en su versión española, que mide las creencias respecto a la sobremedicalización y a la peligrosidad de los medicamentos, donde valores inferiores representaban creencias más deseables en una escala del 4 al 20 (12) (figura 2).

Además, en el cuestionario se preguntaba por características sociodemográficas, y el uso de medicamentos en los tres meses anteriores.

Tabla 1 Áreas de conocimientos y actitudes
ÁREAS DE CONOCIMIENTO
A. ¿Qué es un medicamento?
B. ¿Cuáles son sus condiciones y procesos de uso?
C. ¿Cómo interactúa el medicamento con el individuo y viceversa?
D. ¿Dónde informarse sobre su uso y dónde debe adquirirse?

A. Características del medicamento

1. Concepto de medicamento.

2. Principio activo y nombre comercial, genéricos.

3. Formas farmacéuticas.

4. Símbolos en envases y material de acondicionamiento.

\section{B. Condiciones y procesos de uso}
5. Vias de administración.
6. Condiciones de conservación, almacenamiento, botiquín.
7. Reciclado de los medicamentos, SIGRE, eliminación.

C. Interacciones entre medicamentos y usuarios/pacientes
8. Dosis, pauta y duración de tratamiento.
9. Adherencia, cumplimiento terapéutico, duración del tratamiento.
10. Efectos terapéuticos esperados y no esperados.
11. Interacciones entre medicamentos, entre medicamentos y alimentos, y con el alcohol.
12. Contraindicaciones y situaciones especiales de uso.

D. Fuentes de conocimiento y adquisición de medicamentos. Rol del farmacéutico

13. Información sobre el uso de medicamentos y fuentes de consulta.

14. Regulación de la adquisición y vías para la obtención de medicamentos. Otros canales diferentes a la farmacia.

15. Funciones del farmacéutico comunitario.

Para medir el grado de satisfacción de los alumnos con la intervención educativa se diseñó un breve cuestionario de satisfacción de 3 ítems con preguntas cerradas de elección múltiple de escala de 4 puntos.

Y para el personal docente se diseñó un cuestionario de 3 ítems con preguntas cerradas de elección múltiple (escala Likert de 5 puntos) y 4 ítems con preguntas cerradas dicotómicas.

\section{Plan de trabajo y método}

Desde cada delegación de SEFAC se eligió la provincia donde se llevaría a cabo el proyecto, nombrando a dos coordinadores del mismo en cada provincia. Posteriormente los directores técnicos del proyecto formaron a esos coordinadores provinciales informándoles sobre el proyecto en general, además de proporcionarles todos los protocolos, metodología, materiales, y documentación asociada al estudio, con el objetivo de conseguir que las intervenciones educativas fueran lo más homogéneas posibles. Posteriormente los coordinadores provinciales siguiendo el mismo esquema de trabajo, formaron e informaron en sus respectivas delegaciones a los farmacéuticos comunitarios voluntarios que impartieron las intervenciones educativas.

Se contactó desde cada delegación de SEFAC con los centros educativos necesarios para obtener un tamaño de muestra adecuado, para presentarles el proyecto y pedir su participación, buscando en la medida de lo posible la paridad entre centros urbanos y rurales, y centros privados-concertados, y públicos, aunque no fue un requisito indispensable.

La elección del curso de bachillerato participante y la aleatorización a grupo intervención (GI) y control (GC) se realizó por clase en cada centro educativo por la dirección del centro. En cada provincia se procuró que los grupos control e intervención presentaran tamaños de muestra similares. 


\section{MIRA LA FIGURA QUE APARECE A CONTINUACIÓN}

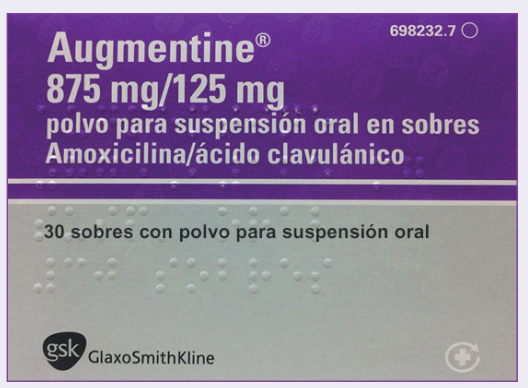

1.1. Indica el principio activo o fármaco del medicamento

1.2. Indica la dosis de principio activo del medicamento

1.3. Indica la forma farmacéutica de este medicamento

1.4. Indica el nombre de la marca registrada de este medicamento

1.5. ¿Es este un medicamento genérico?
Sí
No
No lo sé

\section{MIRA LA FIGURA QUE APARECE A CONTINUACIÓN}

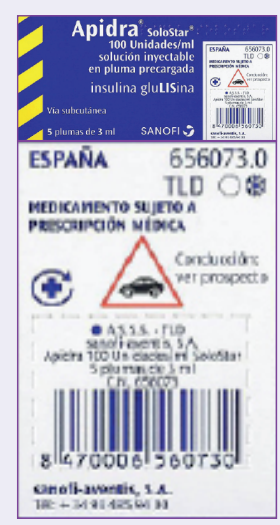

¿Sabrías decir qué significan los siguientes símbolos del envase?

2.1.

A. Medicamento genérico

B. En comprimidos

C. Apto para todas las edades

D. Necesita receta del médico

E. No lo sé

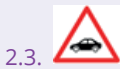

A. No tomar el medicamento si vas a conducir

B. Puede afectar a la capacidad para conducir

C. El medicamento puede dar positivo en el test de control de drogas

D. No llevar el medicamento en el coche

E. No lo sé
2.2.

A. Reciclar en farmacia

B. Venta en farmacia sin receta

C. Consulta al farmacéutico

D. Es un producto farmacéutico

E. No lo sé

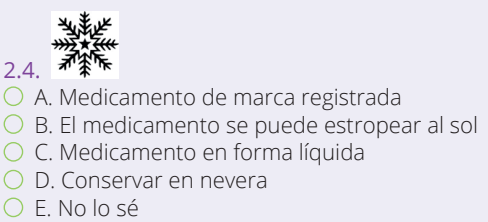

\section{INDICA A QUÉ FORMA FARMACÉUTICA CORRESPONDE CADA IMAGEN}
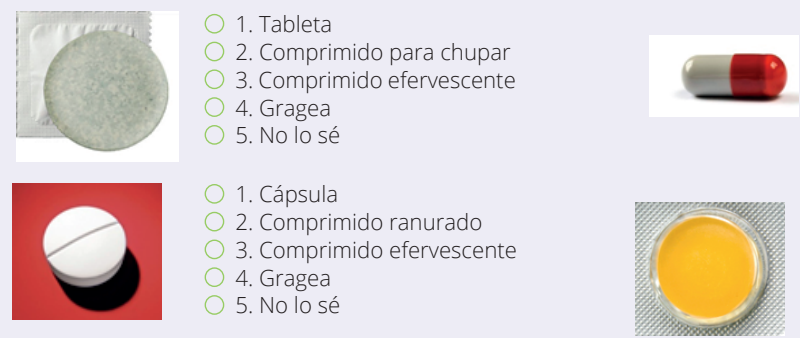

1. Cápsula

2. Comprimido para chupar

3. Tableta

4. Gragea

5. No lo sé

\section{ASOCIA A CADA FORMA FARMACÉUTICA SU VÍA DE ADMINISTRACIÓN}

Pueden repetirse las vías de administración para diferentes formas farmacéuticas: inhalatoria, intramuscular, ocular, oral, rectal, tópica, vaginal.

\begin{tabular}{|c|c|c|c|c|c|c|c|c|}
\hline $\begin{array}{l}\text { Forma } \\
\text { farmacéutica }\end{array}$ & Vía de admin & ración & & & & & & \\
\hline $\begin{array}{l}\text { Ejemplo: } \\
\text { Cápsula }\end{array}$ & Inhalatoria & Intramuscular & Ocular & Oral & Rectal & Tópica & Vaginal & - No lo sé \\
\hline Supositorio & Inhalatoria & Intramuscular & Ocular & Oral & Rectal & Tópica & Vaginal & No lo sé \\
\hline Sobre & Inhalatoria & Intramuscular & Ocular & Oral & Rectal & Tópica & Vaginal & No lo sé \\
\hline Pomada & Inhalatoria & Intramuscular & Ocular & Oral & Rectal & Tópica & Vaginal & No lo sé \\
\hline Óvulo & Inhalatoria & Intramuscular & Ocular & Oral & Rectal & Tópica & Vaginal & No lo sé \\
\hline Jarabe & Inhalatoria & Intramuscular & Ocular & Oral & Rectal & Tópica & Vaginal & No lo sé \\
\hline Inyectable & Inhalatoria & Intramuscular & Ocular & Oral & Rectal & Tópica & Vaginal & No lo sé \\
\hline Gragea & Inhalatoria & Intramuscular & Ocular & Oral & Rectal & Tópica & Vaginal & No lo sé \\
\hline Comprimido & Inhalatoria & Intramuscular & Ocular & Oral & Rectal & Tópica & Vaginal & No lo sé \\
\hline Colirio & Inhalatoria & Intramuscular & Ocular & Oral & Rectal & Tópica & Vaginal & No lo sé \\
\hline Aerosol & Inhalatoria & Intramuscular & Ocular & Oral & Rectal & Tópica & Vaginal & No lo sé \\
\hline
\end{tabular}




\section{PARA LAS SIGUIENTES PREGUNTAS, ¿PODRÍAS SEÑALAR CUÁL ES LA RESPUESTA CORRECTA?}

5.1. Cuando un medicamento produce un efecto no deseado y/o dañino se llama...

A. Interacción

B. Efecto adverso

C. Contraindicación

D. Posología

E. No lo sé

5.2. Cuando una persona no puede tomar un medicamento por una situación personal (por ejemplo, estar embarazada) hablamos de...

A. Interacción

B. Efecto adverso

C. Contraindicación

D. Posología

E. No lo sé

5.3. Cuando el efecto de un medicamento que tomo se modifica por culpa de otro medicamento o alimento que tomo se llama...

A. Interacción

B. Efecto adverso

C. Contraindicación

D. Posología

E. No lo sé

5.4. Cuando el médico pauta Paracetamol 500 mg 3 veces al día, ¿qué hay que hacer?

A. Tomar una pastilla de $500 \mathrm{mg}$ al levantarse, al mediodía y antes de acostarse

B. Tomar una pastilla de $500 \mathrm{mg}$ con el desayuno, la comida y la cena

C. Tomar una pastilla de $500 \mathrm{mg}$ cada 8 horas

D. Tomar una pastilla de $500 \mathrm{mg}$ a las $9 \mathrm{~h}$, las $15 \mathrm{~h}$ y las $21 \mathrm{~h}$

E. No lo sé

5.5. ¿Durante cuánto tiempo debe tomarse un antibiótico?

A. Hasta que desaparece la fiebre

B. Hasta que desaparece el dolor o la inflamación

C. El indicado por su médico

D. Durante siete días

E. No lo sé
5.6. ¿Cuándo puedes tomar un medicamento sin consultar a un profesional sanitario?

A. Nunca, siempre deberá estar indicado por un profesional sanitario

B. Cuando es un medicamento que ya te han indicado en el pasado

C. Cuando se lo dieron a un familiar o amigo para el mismo problema

D. Cuando es un medicamento de uso común como e ibuprofeno o el paracetamol

E. No lo sé

5.7. ¿Cómo deben tomarse los medicamentos que se toman por vía oral?

A. Siempre con alimentos o durante las comidas

B. Dependerá de cada medicamento en concreto

C. Nunca con alimentos o durante las comidas

D. O una hora antes o dos después de las comidas

E. No lo sé

5.8. ¿Dónde deben guardarse los medicamentos?

A. Preferiblemente en la cocina

B. Preferiblemente en el baño

C. Preferiblemente en una habitación o en el salón

D. Pueden guardarse en cualquier sitio

E. No lo sé

5.9. ¿Cómo se eliminan los medicamentos que sobran o que han caducado?

A. Se tiran a la basura

B. Se tiran por el inodoro o water

C. Se tiran en el contenedor de reciclaje de plástico y papel

D. Se llevan a la farmacia

E. No lo sé

Figura 1 (Continuación)

A continuación, aparece una lista de afirmaciones que otras personas han declarado sobre los medicamentos en general. Por favor, señala en qué medida estás de acuerdo o en desacuerdo con ellas marcando la casilla apropiada. No hay respuestas correctas ni incorrectas. Estamos interesados en tus opiniones personales.

\begin{tabular}{|c|c|c|c|c|c|}
\hline & $\begin{array}{l}\text { Totalmente en } \\
\text { desacuerdo }\end{array}$ & En desacuerdo & $\begin{array}{l}\text { Ni de acuerdo ni } \\
\text { en desacuerdo }\end{array}$ & De acuerdo & $\begin{array}{l}\text { Totalmente de } \\
\text { acuerdo }\end{array}$ \\
\hline $\begin{array}{l}\text { 1. Los médicos prescriben demasiados } \\
\text { medicamentos }\end{array}$ & O & O & O & O & O \\
\hline $\begin{array}{l}\text { 2. La gente que toma medicamentos debería dejar su } \\
\text { tratamiento durante algún tiempo de vez en cuando }\end{array}$ & 0 & 0 & ○ & 0 & ○ \\
\hline 3. La mayoría de medicamentos crean adicción & 0 & 0 & 0 & 0 & 0 \\
\hline $\begin{array}{l}\text { 4. Los remedios naturales son más seguros que } \\
\text { los medicamentos }\end{array}$ & O & 0 & O & 0 & O \\
\hline 5. Los medicamentos hacen más mal que bien & O & 0 & O & 0 & 0 \\
\hline $\begin{array}{l}\text { 6. Todos los medicamentos son venenosos } \\
\text { (tóxicos) }\end{array}$ & 0 & ○ & O & O & 0 \\
\hline $\begin{array}{l}\text { 7. Los médicos confían demasiado en los } \\
\text { medicamentos }\end{array}$ & 0 & O & O & O & O \\
\hline $\begin{array}{l}\text { 8. Si los médicos tuvieran más tiempo para los } \\
\text { pacientes recetarían menos medicamentos }\end{array}$ & O & O & O & 0 & 0 \\
\hline
\end{tabular}

Figura 2 Cuestionario "Beliefs about Medicines Questionnaire" (BMQ) versión española 


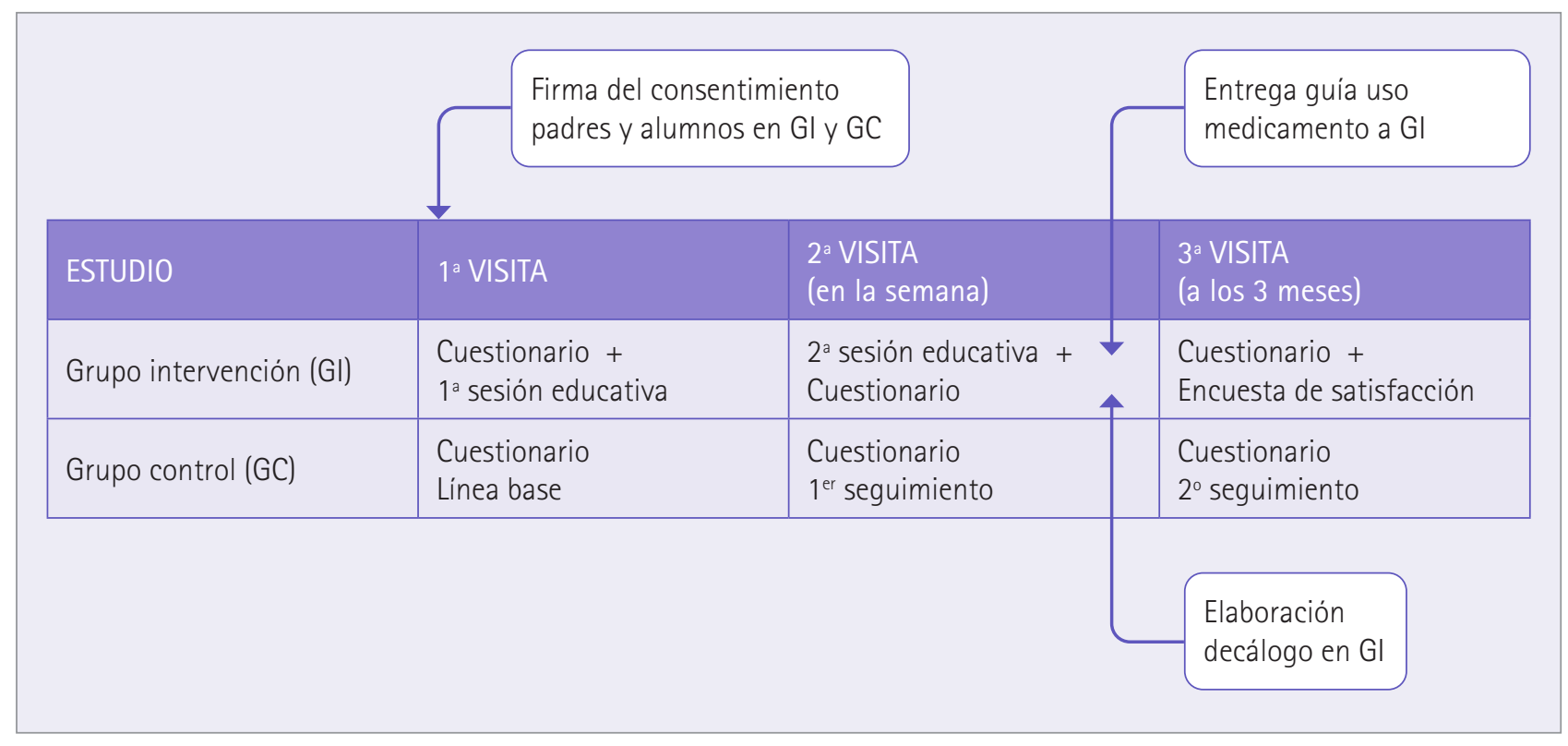

Figura 3 Esquema del plan de trabajo general para los centros educativos

Metodología de las intervenciones educativas

Días antes de la intervención se entregó en clase a los alumnos el consentimiento informado para que fuera firmado por sus padres o tutores legales, y otro adicional donde se les proporcionaba información sobre el proyecto y las instrucciones necesarias para poder participar en el mismo.

Las intervenciones educativas se aplicaron a los alumnos del grupo intervención. Se les pidió que cada uno de ellos llevara el día de la sesión formativa un medicamento (envase vacío con el prospecto) que tuvieran en casa con el que hubieran sido tratados en alguna ocasión, preferentemente uno de uso común. Durante las intervenciones educativas se utilizaron estos medicamentos como parte práctica del aprendizaje. Las intervenciones se realizaron preferentemente en la misma aula donde recibían clases normalmente los alumnos, dependiendo de la disponibilidad de cada centro, deseando que fueran grupos pequeños de unos 20-25 alumnos. La intervención educativa se realizó en dos sesiones de 45 minutos cada una, ya que se consideró que alargar la duración de las mismas sería contraproducente en aras de conseguir los objetivos deseados.

Los farmacéuticos voluntarios se valieron también en las sesiones formativas de diferentes envases y formas farmacéuticas de medicamentos para trabajar pedagógicamente con ejemplos prácticos con la finalidad de mejorar el aprendizaje.

Después de las dos sesiones formativas, y como trabajo para los alumnos se les pidió que entre todos elaborasen un decálogo con los aspectos que ellos creyeran más importantes sobre el uso y manejo de los medicamentos. De esta manera se reforzó el mensaje de las intervenciones educativas. En el desarrollo de las mismas se priorizó la participación activa del alumno, huyendo de la fórmula de clase magistral, con el objetivo final de que los alumnos adoptasen actitudes e interiorizasen conocimientos sobre el uso adecuado de los medicamentos.

A los alumnos de ambos grupos, intervención y control, se les instruyó también en la cumplimentación de los cuestionarios utilizados en este estudio.

El esquema del plan de trabajo general para cada centro educativo se refleja en la figura 3. Una vez finalizado el estudio y por cuestiones éticas se realizó la intervención educativa al GC y también se entregó la guía práctica para el uso adecuado de los medicamentos a los alumnos.

Así pues, los alumnos rellenaron los dos cuestionarios en tres ocasiones: antes de iniciar la primera sesión educativa, la llamada "línea base", al finalizar la segunda sesión educativa ("primer seguimiento") y al cabo de tres meses de haber completado por primera vez los cuestionarios ("segundo seguimiento"). En el segundo seguimiento, al finalizar el último cuestionario, los participantes del grupo intervención también valoraron su satisfacción con la actividad educativa en su conjunto.

Así mismo, los profesores responsables de la actividad en el centro al finalizar valoraron su satisfacción con el proyecto.

\section{Variables de estudio}

Las variables principales fueron, el conocimiento básico sobre medicamentos y las creencias y actitudes respecto a la sobremedicalización y respecto a la peligrosidad de los medicamentos.

Las variables secundarias fueron edad, sexo, si habían utilizado medicamentos los últimos tres meses o no, medicamento usado, provincia, y centro educativo.

\section{Análisis estadísticos}

Se presentan datos descriptivos para las variables sociodemográficas y clínicas: frecuencias y proporciones para las variables categóricas y medias y errores estándares para las variables continuas. Los datos se presentan por separado estratificando la muestra por grupo intervención (GI) o control (GC). Las diferencias entre ambos grupos en línea base se probaron mediante modelos multinivel de efectos aleatorios para medidas repetidas, considerándose tres niveles: la provincia, el centro educativo y el estudiante. 
Las variables dependientes del estudio fueron: el nivel de conocimiento básico sobre el uso del medicamento, de creencia respecto a la sobremedicalización y de creencia respecto a la peligrosidad de los medicamentos. Para evaluar el efecto de las intervenciones educativas en las variables dependientes, siguiendo una estrategia de análisis por intención de tratar, se llevaron a cabo modelos multinivel de efectos aleatorios para medidas repetidas. Se consideraron cuatro niveles: la provincia, el centro educativo, el estudiante y el tiempo (línea base, después de la intervención y 3 meses después). Para evaluar el efecto de la intervención sobre las variables, se testeó el efecto de la interacción grupo por tiempo en cada una de las variables dependientes. Los modelos se ajustaron por aquellas variables que mostraron diferencias estadísticamente significativas entre grupos en línea base. Se consideró que la intervención había tenido un impacto estadísticamente significativo cuando la interacción grupo por tiempo fue estadísticamente significativa.

A partir de los modelos se calcularon niveles medios de conocimiento y creencias y se compararon los resultados obtenidos entre ambos grupos (intervención o control) para toda la muestra o según el nivel inicial de conocimiento y creencias. Para ello, se dividió a la muestra en dos: aquellos estudiantes que presentaban una puntuación en línea base por encima de la mediana en la variable dependiente y aquellos que presentaban una puntuación por debajo de la mediana.

Los modelos multinivel ajustados se utilizaron para estimar una representación gráfica lineal de la evolución en las variables dependientes.

Para todos los análisis se utilizó el software estadístico STATA 13 con un nivel de significación de $\mathrm{p}<0,05$.

\section{Resultados}

Características de la muestra

El proyecto se desarrolló en 17 provincias, una por comunidad autónoma durante los cursos 2018/19 y 2019/20. En él colaboraron 336 farmacéuticos comunitarios con una media de 20 voluntarios por provincia. Participaron 232 centros educativos, de media 14 centros por provincia, distribuidos de la siguiente manera; $40 \%$ en capital de provincia, $41 \%$ en población urbana y 19\% en rural (<10.000 habitantes), siendo de titularidad pública el $62 \%$ de los centros.

Participaron un total de 15.711 alumnos, con una media de 924 por provincia que sumaron un total de 298 grupos de intervención y otros 283 de control, resultando que el 90\% del GI y el 75\% del GC pertenecían a $1^{\circ}$ bachillerato.

Finalmente, después de descartar los registros con incidencias relevantes para su análisis se analizaron las respuestas de 7.203 alumnos del GI y 6.652 del GC, que significaban un $89 \%$ y $88 \%$ respecto a la participación inicial.

Características sociodemográficas

La tabla 2 muestra que cerca del $54 \%$ de los participantes eran de sexo femenino. La edad media fue próxima a los 16,5 años (15-20). El 85\% había utilizado medicamentos en los tres meses anteriores, un 16\% había utilizado antibióticos, un $20 \%$ ibuprofeno $400 \mathrm{mg}$, un $41 \%$ ibuprofeno $600 \mathrm{mg}$ y un $38 \%$ paracetamol, entre otros.

\section{Tabla 2 Caracteristicas sociodemográficas de la muestra}

\begin{tabular}{|c|c|c|c|}
\hline Variable & $\begin{array}{c}\text { Grupo } \\
\text { control } \\
\text { n (\%) }\end{array}$ & $\begin{array}{c}\text { Grupo } \\
\text { intervención } \\
n(\%)\end{array}$ & $\mathrm{p}$-valor \\
\hline \multicolumn{4}{|l|}{ Sexo } \\
\hline Masculino & $3.055(45,9)$ & $3.274(45,4)$ & \multirow{2}{*}{0,444} \\
\hline Femenino & $3.597(54,1)$ & $3.929(54,6)$ & \\
\hline Edad (rango 14-20) [Media (DE)] & $16,6(0,01)$ & $16,4(0,01)$ & $<0,001$ \\
\hline \multicolumn{4}{|l|}{ Detalle de la distribución por edades } \\
\hline 14 años & $0(0,00)$ & $1(0,01)$ & \\
\hline 15 años & $778(11,7)$ & $1.163(16,2)$ & \\
\hline 16 años & $2.587(38,9)$ & $3.107(43,1)$ & \\
\hline 17 años & $2.343(35,2)$ & $2.243(31,1)$ & \\
\hline 18 años & $720(10,8)$ & $511(7,1)$ & \\
\hline 19 años & $127(1,9)$ & $101(1,4)$ & \\
\hline 20 años & $97(1,5)$ & $77(1,1)$ & \\
\hline \multicolumn{4}{|l|}{ Medicación reciente } \\
\hline No & $823(12,4)$ & $851(11,8)$ & \multirow{3}{*}{0,381} \\
\hline Si & $5.620(84,6)$ & $6.126(85,1)$ & \\
\hline No lo sé & $204(3,1)$ & $222(3,1)$ & \\
\hline \multicolumn{4}{|l|}{ Medicamentos más frecuentes } \\
\hline Antibiótico & $1.084(16,3)$ & $1.194(16,6)$ & 0,678 \\
\hline Ibuprofeno 400 mg & $1.298(19,5)$ & $1.480(20,6)$ & 0,103 \\
\hline Ibuprofeno $600 \mathrm{mg}$ & $2.781(41,8)$ & $2.888(40,1)$ & $<0,05$ \\
\hline \multirow[t]{2}{*}{ Paracetamol } & $2.597(39,0)$ & $2.714(37,7)$ & 0,094 \\
\hline & Media (DE) & Media (DE) & \\
\hline Nivel conocimiento (rango 0-10) & $5,5(0,02)$ & $5,5(0,02)$ & 0,377 \\
\hline BMO sobremedicalización (rango 4-20) & $12,2(0,03)$ & $12,0(0,03)$ & $<0,001$ \\
\hline BMO peligrosidad (rango 4-20) & $9,5(0,03)$ & $9,4(0,03)$ & 0,066 \\
\hline
\end{tabular}


Tabla 3 Impacto de la intervención educativa en el conocimiento

\begin{tabular}{|c|c|c|c|}
\hline Característica & Beta & $\begin{array}{c}\text { Intervalo de } \\
\text { confianza (95\%) }\end{array}$ & p-valor \\
\hline Constante & 5,48 & $5,39 \mid 5,57$ & $<0,001$ \\
\hline \multicolumn{4}{|l|}{ Grupo } \\
\hline Control & Ref. & & \\
\hline Intervención & $-0,09$ & $-0,14 \mid-0,04$ & $<0,005$ \\
\hline \multicolumn{4}{|l|}{ Observación } \\
\hline Linea base & Ref. & & \\
\hline Primer seguimiento & 0,35 & $0,31 \mid 0,38$ & $<0,001$ \\
\hline Segundo seguimiento & 0,44 & $0,40 \mid 0,48$ & $<0,001$ \\
\hline \multicolumn{4}{|l|}{ Interacción Grupo x Observación } \\
\hline Control x Línea base & Ref. & & \\
\hline Interv. x Primer seguimiento & 1,26 & $1,21 \mid 1,31$ & $<0,001$ \\
\hline Interv. x Segundo seguimiento & 0,97 & $0,92 \mid 1,02$ & $<0,001$ \\
\hline
\end{tabular}

Análisis realizado con 13.693 casos con información válida en la variable de conocimiento. Beta $=$ diferencia media en la variable dependiente.

\section{Efecto de las intervenciones} educativas en el conocimiento sobre medicamentos

En la tabla 3 se muestra el impacto de la intervención en el nivel de conocimiento sobre medicamentos a través de un modelo multinivel de efectos aleatorios para medidas repetidas. La interacción entre grupo y tiempo fue estadísticamente significativa, lo que indica que existen diferencias en la evolución del nivel medio de conocimiento entre el grupo control e intervención y, por tanto, que la formación mejoró el conocimiento sobre medicamentos de los estudiantes.

En la figura 4 se presentan las evoluciones de las medias de nivel de conocimiento, extraídas del modelo multinivel, para los grupos de control e intervención a través de las distintas observaciones. En el grupo intervención, el nivel de conocimiento medio fue de 5,4 en línea base, 7,0 en el primer seguimiento y 6,8 en el segundo seguimiento (resultando una mejora media de 1,4 puntos), mientras que en el grupo de control fue del 5,5 en la línea base, 5,8 en el primer seguimiento y 5,9 en el segundo.

Por otra parte, si evaluamos la evolución del nivel medio de conocimiento sobre medicamentos en los grupos intervención y control estratificando la muestra según el nivel medio de conocimiento en línea base, observamos que la interacción entre grupo y tiempo fue estadísticamente significativa tanto en el grupo de conocimiento alto en línea base como en el de conocimiento bajo, lo que indica que la formación mejoró el conocimiento sobre medicamentos de los estudiantes independientemente del nivel de conocimiento en línea base.

Así, en el grupo de conocimiento alto en línea base los participantes del grupo intervención partieron de un nivel medio de conocimiento de 6,5, que aumentó a 7,5 en el primer seguimiento y descendió a 7,3 en el segundo (resultando una mejora media de 0,8 puntos). Los participantes del grupo control que partieron de un nivel medio de conocimiento de 6,5 no presentaron variaciones ni en el primer ni en el segundo seguimiento. Mientras que en el grupo de conocimiento bajo en línea base los participantes del grupo de intervención presentaron un conocimiento medio de 4,3 en la línea base, 6,5 en el primer

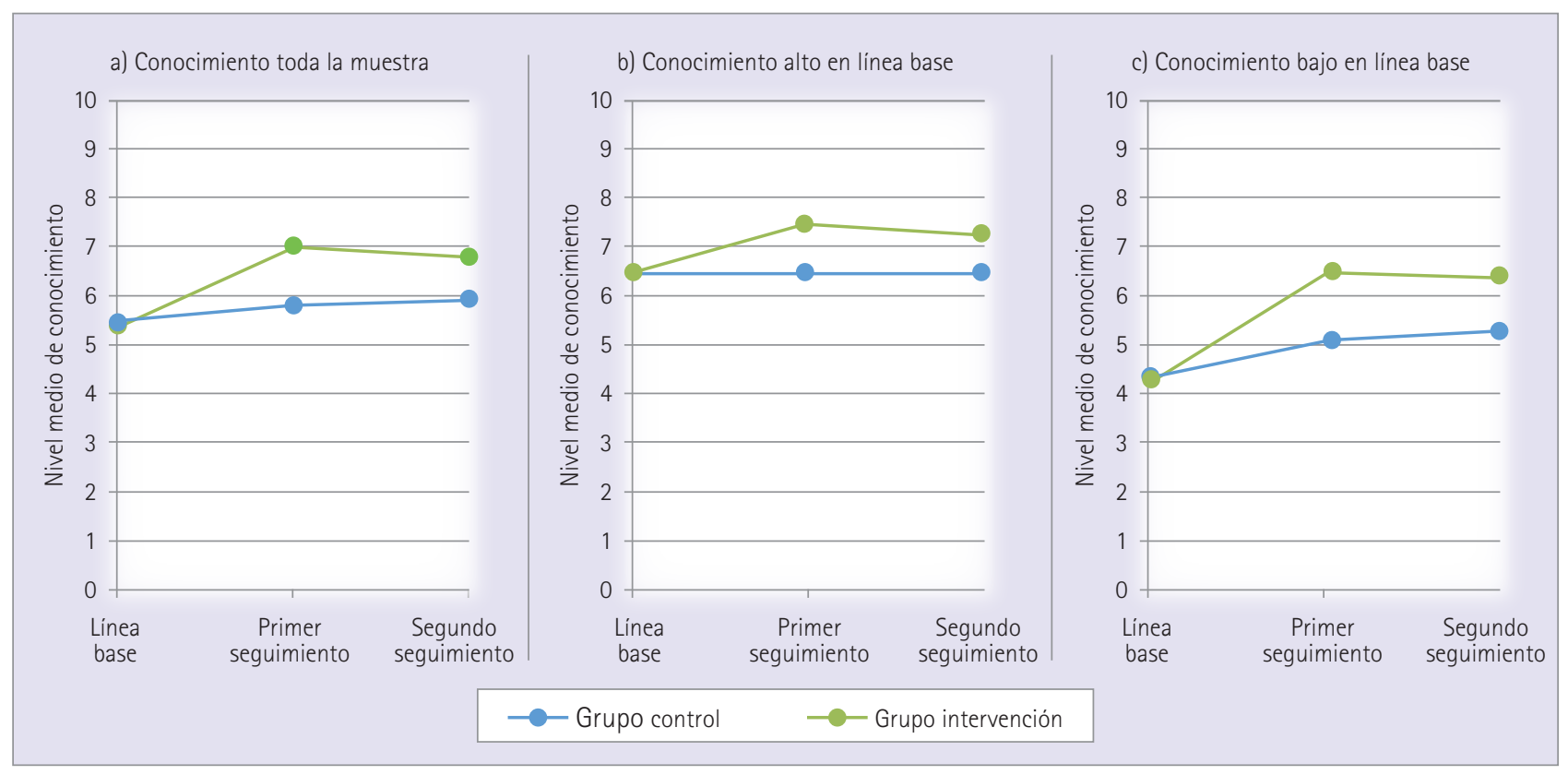

Figura 4 Efecto de la intervención educativa en el conocimiento sobre medicamentos para el total de la muestra (a) y según el nivel de conocimientos en línea base* (b: nivel alto, c: nivel bajo)

* El nivel de conocimiento en línea base se consideró alto cuando el alumno puntuó por encima de la mediana y bajo cuando puntuó por debajo. 
seguimiento y 6,4 en el segundo (resultando una mejora media de 2,1 puntos). Los participantes del grupo control que partieron de un nivel medio de conocimiento de 4,4 aumentaron a 5,1 en el primer seguimiento y a 5,3 en el segundo. Lo que nos permite afirmar que el impacto de la intervención sobre el conocimiento fue superior cuando el nivel de conocimiento en línea base era más bajo.
En la figura 5 se aprecia el impacto de las intervenciones educativas desglosado por provincias participantes en el estudio. En todos los casos la interacción entre grupo y

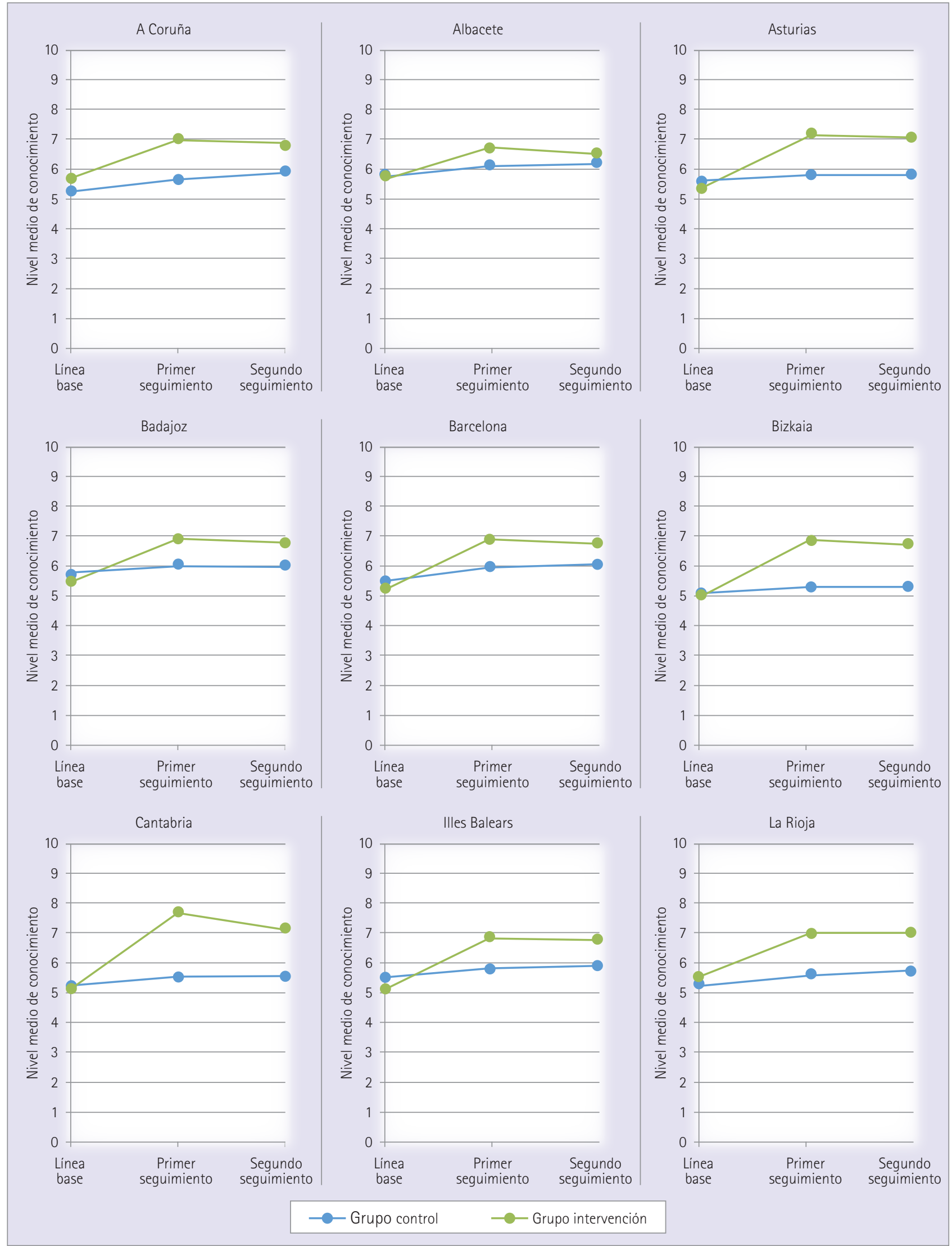

Figura 5 Efecto de la intervención educativa en el conocimiento sobre medicamentos por provincias 


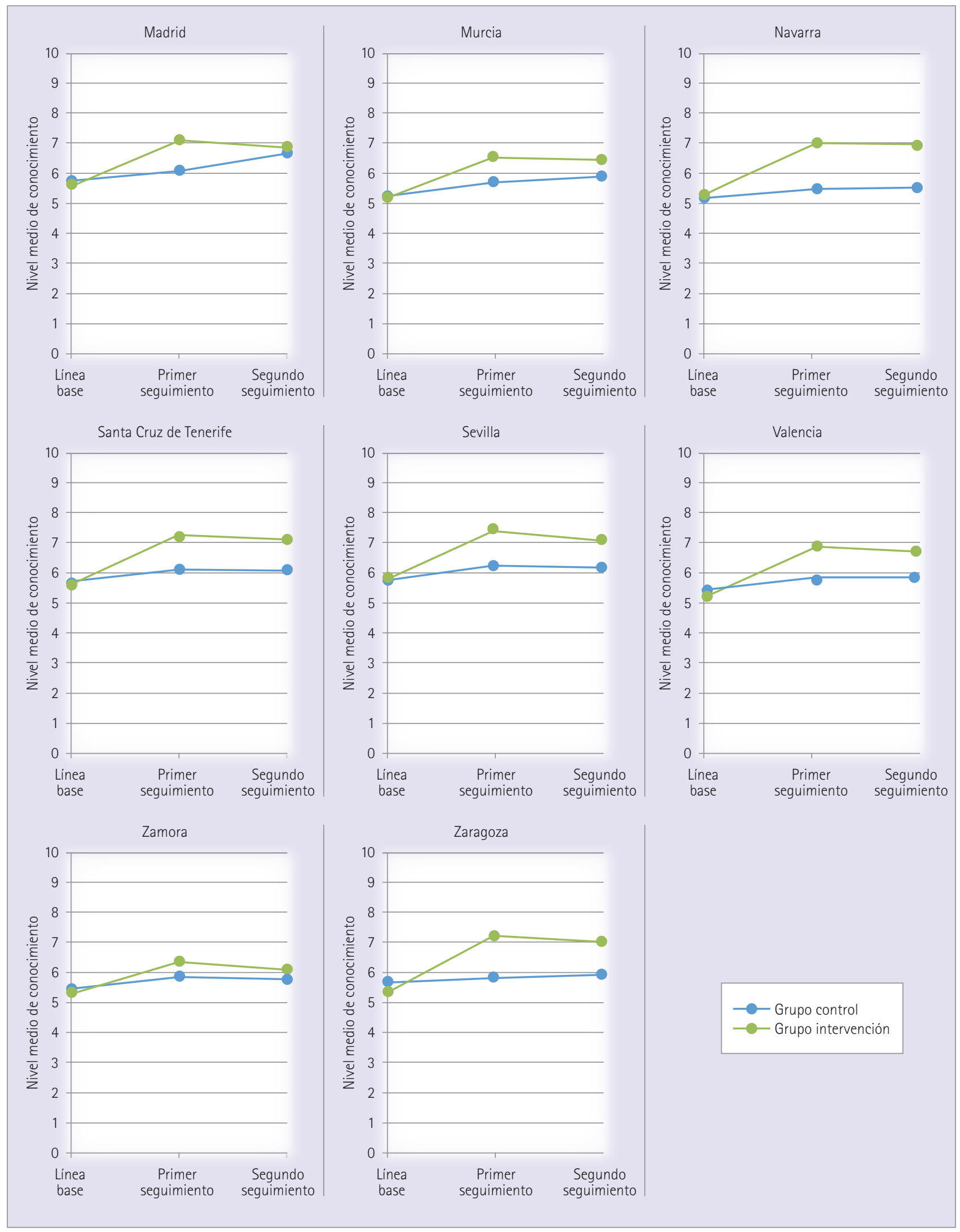

Figura 5 (Continuación)

tiempo fue estadísticamente significativa.

Se recogieron 247 decálogos realizados en clase por los alumnos de los GI en días posteriores a la segunda sesión educativa.
Efecto de las intervenciones educativas en la creencia respecto a la sobremedicalización y peligrosidad de los medicamentos

Para evaluar la evolución en el nivel medio de creencias y actitudes se utilizó el "Beliefs about Medicines Questionnaire” (BMQ) en su versión española con una escala 4-20, donde valores inferiores indican creencias más positivas. Los resultados generales se reflejan en la figura 6 . 


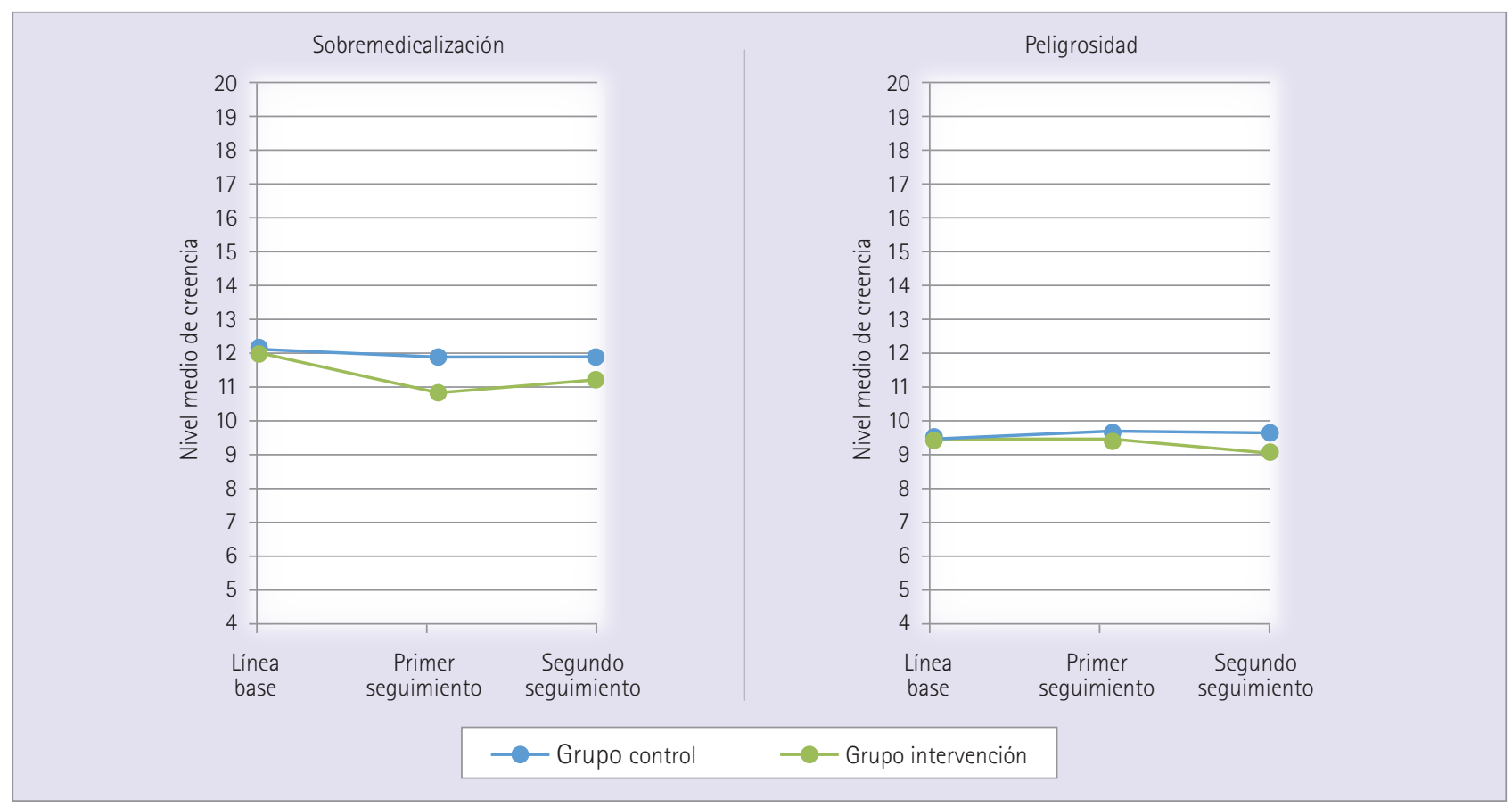

Figura 6 Efecto de la intervención educativa en el nivel de creencia respecto a la sobremedicalización y a la peligrosidad de los medicamentos para el total de la muestra

Respecto a las creencias en sobremedicalización los resultados del cuestionario para el GI fueron de 12,0 en línea base, de 10,8 en primer seguimiento y 11,2 para el segundo, resultando una mejora media final de 0,8 puntos, siendo más evidente el efecto de la intervención en el grupo con creencias más negativas en línea base, como se refleja en la figura 7. En el GC fueron 12,2 11,9 - 11,9, respectivamente.

Respecto a las creencias sobre peligrosidad los resultados del cuestionario para el GI fueron en línea base de 9,4, en primer seguimiento 9,4, y 9,1, en segundo seguimiento con una mejora media de 0,3 puntos. En GC fueron 9,5-9,7 - 9,6, respectivamente.
La interacción grupo tiempo resultó estadísticamente significativa en los dos casos, lo que indica que existen diferencias significativas en la evolución entre GI y GC y, por tanto, que la formación mejoró el nivel de creencias y actitudes de los estudiantes respecto a la sobremedicalizacion y la peligrosidad de los medicamentos.

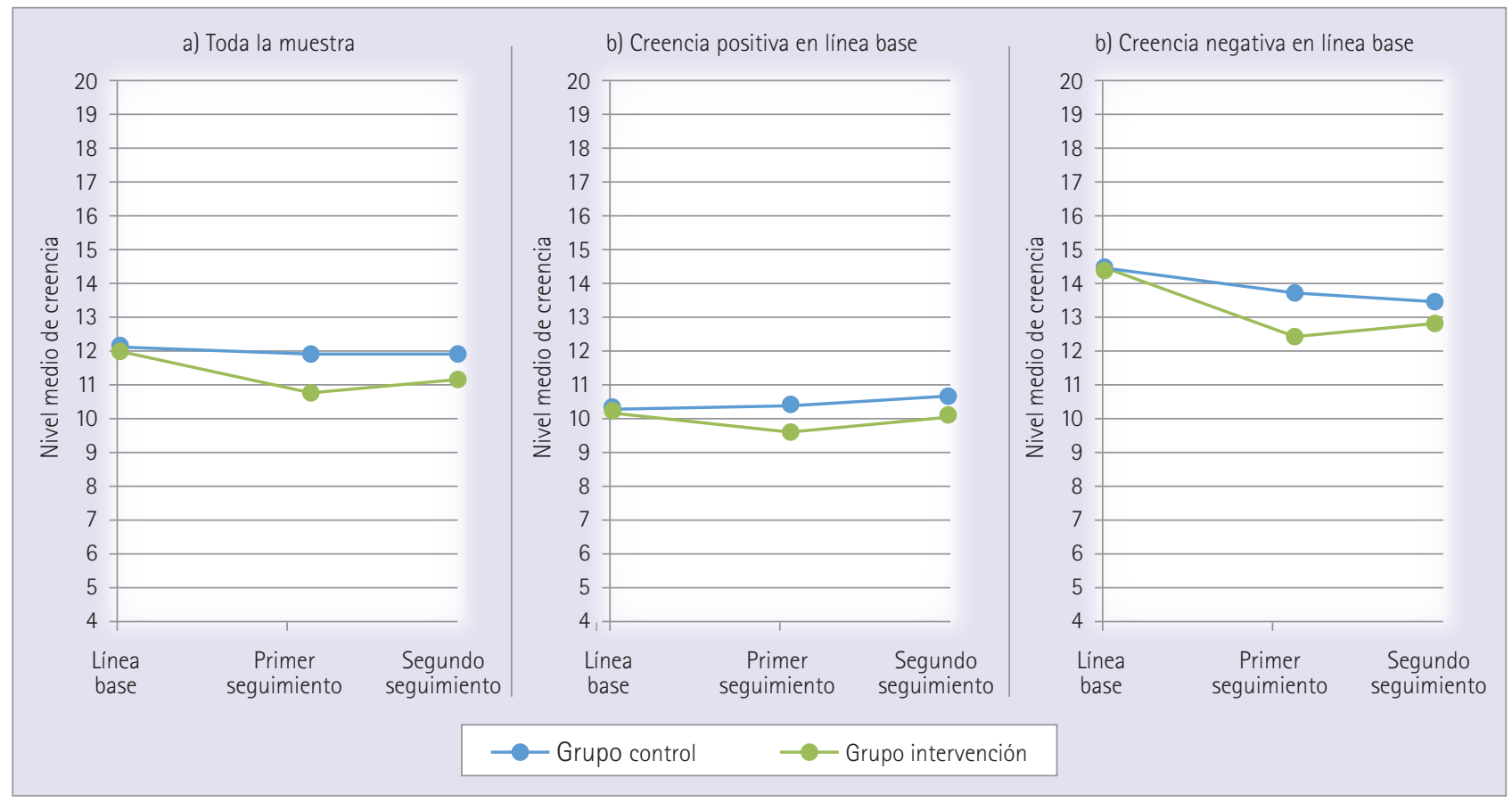

Figura 7 Efecto de la intervención educativa en la creencia respecto a la sobremedicalización para el total de la muestra (a) y según el nivel de creencia en línea base* (b: positivo, c: negativo).

* El nivel de creencia en línea base se consideró positivo cuando el alumno puntuó por debajo de la mediana y negativo cuando puntuó por encima. 
Satisfacción con la actividad educativa

En el segundo seguimiento, los participantes del grupo de intervención valoraron su satisfacción con la actividad. Se analizaron los resultados de aquellos alumnos que acudieron, como mínimo, a una de las dos sesiones previas $(n=5.795)$. Como se puede apreciar en la tabla 4, en términos generales el $84 \%$ de los alumnos encontró bastante o muy interesante la actividad educativa. El $80 \%$ de los alumnos consideró que había mejorado su conocimiento sobre el uso del medicamento y el $86 \%$ lo recomendaría a un compañero.

Al finalizar el proyecto en el centro, los profesores responsables de la actividad valoraron su satisfacción. Como se puede apreciar en la tabla 5, en general el 95\% se mostró satisfecho con el proyecto e interesado en repetir la actividad educativa el próximo curso.

Tabla 4 Satisfacción de los alumnos con la actividad educativa

\section{n (\%)}

¿Te ha parecido interesante

el estudio del medicamento?

\begin{tabular}{|l|c|}
\hline Nada & $177(3,1)$ \\
\hline Poco & $724(12,5)$ \\
\hline Bastante & $3.010(51,9)$ \\
\hline Mucho & $1.884(32,5)$ \\
\hline No responde & $0(0,0)$ \\
\hline
\end{tabular}

¿Ha mejorado tu conocimiento sobre el uso del medicamento?

\begin{tabular}{|l|c|}
\hline Nada & $146(2,5)$ \\
\hline Poco & $984(17,0)$ \\
\hline Bastante & $3.095(53,4)$ \\
\hline Mucho & $1.567(27,0)$ \\
\hline No responde & $3(0,1)$ \\
\hline $\begin{array}{l}\text { ¿Le recomendarias esta actividad a } \\
\text { tus compañeros? }\end{array}$ \\
\hline Nada & $195(3,4)$ \\
\hline Poco & $609(10,5)$ \\
\hline Bastante & $2.675(46,1)$ \\
\hline Mucho & $2.298(39,7)$ \\
\hline No responde & $18(0,3)$ \\
\hline
\end{tabular}

Tabla 5 Satisfacción del personal docente con la actividad educativa

n (\%)

¿Considera adecuada la metodología?

\begin{tabular}{|c|c|}
\hline Nada & $0(0,0)$ \\
\hline Poco & $0(00)$ \\
\hline Aceptable & $5(3,3)$ \\
\hline Bastante & $58(38,4)$ \\
\hline Mucho & $88(58,3)$ \\
\hline No responde & $0(0,0)$
\end{tabular}

¿Considera que la sesión educativa está ajustada al nivel de los alumnos?

\begin{tabular}{|l|c|}
\hline Nada & $0(0,0)$ \\
\hline Poco & $1(0,7)$ \\
\hline Aceptable & $1(0,7)$ \\
\hline Bastante & $47(31,1)$ \\
\hline Mucho & $102(67,5)$ \\
\hline No responde & $0(0,0)$ \\
\hline
\end{tabular}

¿Considera oportuna la documentación que se entrega al alumno al final de la charla? "Guía práctica para el uso adecuado de los medicamentos"

\begin{tabular}{|l|c|}
\hline Nada & $1(0,7)$ \\
\hline Poco & $0(0,0)$ \\
\hline Aceptable & $2(1,3)$ \\
\hline Bastante & $29(19,2)$ \\
\hline Mucho & $116(76,8)$ \\
\hline No responde & $3(2,0)$
\end{tabular}

¿Considera que $1^{\circ}$ bachiller es el curso idóneo para impartir la actividad?

\begin{tabular}{|l|c|}
\hline No & $5(3,3)$ \\
\hline Si & $144(95,4)$ \\
\hline No responde & $2(1,3)$ \\
\hline
\end{tabular}

¿Considera que $2^{\circ}$ bachiller es el curso idóneo para impartir la actividad?

\begin{tabular}{|l|c|}
\hline No & $80(53,0)$ \\
\hline Si & $50(33,1)$ \\
\hline No responde & $21(13,9)$ \\
\hline Si no es así, ¿podría indicar cuál? & $31(20,6)$ \\
\hline $4^{\circ}$ ESO & $6(3,9)$ \\
\hline $3^{\circ}$ ESO &
\end{tabular}

¿Estaría interesado en repetir las sesiones formativas el próximo curso?

\begin{tabular}{|l|c|}
\hline No & $2(1,3)$ \\
\hline Si & $147(97,4)$ \\
\hline No responde & $2(1,3)$ \\
\hline La actividad, ¿ha mejorado su conocimiento sobre al uso de los medicamentos? \\
\hline No & $2(1,3)$ \\
\hline Si & $144(95,4)$ \\
\hline No responde & $5(3,3)$ \\
\hline
\end{tabular}




\section{Discusión}

\section{Antecedentes}

Es obvio que la utilización de un cuestionario de elaboración propia dificulta la comparación de los resultados con otros trabajos que también evalúan conocimientos, creencias y opiniones sobre medicamentos en general. Existen estudios que han perseguido objetivos muy similares a los nuestros, pero o bien la población a estudio era diferente en términos sociodemográficos (13), o bien los cuestionarios utilizados diferían en su diseño y escala como ocurre con el estudio de Puig Soler et al. (10). En otros casos las variables a estudio diferían de las nuestras, aunque la población a estudio sí que podría haber sido comparable (14-15). Por otra parte, otros cuestionarios existentes en nuestro país como el de García Delgado (16), o a nivel internacional (17), abordan el conocimiento específico de la propia medicación de cada paciente, y no evalúan los conocimientos de conceptos, creencias y actitudes generales sobre el proceso de uso de medicamentos en general. Esos cuestionarios permiten establecer un marco de intervención directa sobre cada paciente en concreto, pero no para obtener una visión global de los conocimientos y creencias poblacionales. Por todos esos motivos, el único estudio mínimamente comparable es el que realizó Joaquina Huarte Royo, farmacéutica comunitaria en Pamplona, durante el curso escolar 2015-16 en centros escolares de Navarra $(n=244)$, con el objetivo de determinar la necesidad de realizar intervenciones educativas sobre el correcto uso de los medicamentos en adolescentes de bachillerato, así como demostrar que estas intervenciones repercutian de forma positiva en el adolescente aumentando sus conocimientos y mejorando sus actitudes frente al uso de medicamentos $(18,19)$. Lo hizo con una metodología similar a este estudio, pero ni el diseño, ni el tratamiento estadístico o los cuestionarios utilizados son los mismos que hemos utilizado en esta ocasión, por lo que se hace difícil establecer posibles comparaciones a nivel de resultados. Aún y así, es interesante resaltar que en ese estudio se observó un porcentaje medio de aciertos en el cuestionario de conocimiento del correcto uso general del medicamento de un 55,3\% $(\mathrm{DE} 13,21)(18)$ previo a las intervenciones educativas, que se elevó al
67,3\% (DE 14,54) después de las intervenciones educativas, siendo significativa la diferencia del $12 \%(\mathrm{p}<0,001)$. Además, casi el 80\% de los alumnos valoró el estudio bastante o muy interesante y el 90,6\% de los padres se mostró bastante o muy satisfecho con la actividad (19), resultados muy en la línea de los obtenidos en este estudio.

\section{Fortalezas y limitaciones}

Entre las fortalezas de este estudio se ha de destacar que el proyecto ConóceMe es un ensayo clínico con un diseño controlado aleatorizado, multicéntrico y de ámbito nacional, realizado en todas las comunidades autónomas, con un elevado número de participantes, lo que aumenta el rigor y permite la obtención de resultados de elevada validez externa sobre la efectividad de las intervenciones. No obstante, existen algunas limitaciones que deberían ser tenidas en cuenta. Los centros participantes se han seleccionado siguiendo diversos criterios de conveniencia, el más importante fue la decisión de participar de forma voluntaria en este estudio por parte de la dirección de los centros; es decir, no de forma totalmente aleatoria, por lo que podría afectar a la validez externa. Sin embargo, se han incluido un gran número de centros en distintas provincias y distintos entornos, rural, urbano, publico, concertado, así como un gran número de participantes, lo que disminuiría el impacto de esta limitación. En segundo lugar, cabría la pequeña posibilidad de que se hubiera podido producir un fenómeno de contaminación cruzada en los alumnos del grupo control. En tercer lugar, no se han tenido en cuenta variables socioeconómicas ni otros factores, como si el grupo intervención era un bachiller de ciencias o letras, que podrían tener un impacto en los resultados obtenidos, aunque en un principio el gran tamaño muestral y la diversidad de los centros participantes minimiza estos posibles sesgos. Por otro lado, el hecho de diseñar un estudio con aleatorización real debería haber garantizado una distribución equilibrada de estas variables entre los grupos control e intervención.

En cuanto a los registros y resultados obtenidos en este estudio, a la pregunta que se hizo a los adolescentes sobre si habian tomado medicamentos los tres meses anteriores al estudio, y si era así, cuáles eran esos medicamentos, llama la atención que el porcentaje de alumnos que habían tomado medicamentos los tres meses anteriores fuera tan elevado (85\%), pero aún más que entre esos medicamentos se encontrasen los antibióti$\cos (16 \%)$ y el ibuprofeno de $600 \mathrm{mg}$ (41\%). Lo más llamativo es la proporción de los medicamentos mencionados, que confirma la sospecha del gran desconocimiento de las indicaciones o del correcto uso de estos medicamentos, que no deberían tener un uso tan común. Normalmente estos medicamentos son parte de los botiquines caseros como consecuencia de tratamientos no finalizados o de dosis sobrantes, y luego son utilizados de forma inadecuada por los adolescentes, en muchos casos siguiendo las recomendaciones de familiares y/o amigos y no de profesionales sanitarios. En definitiva, estos registros avalan la hipótesis de que hay que potenciar la educación sobre el uso racional de los medicamentos en estos colectivos.

En cualquier caso, se ha de constatar que los resultados del presente estudio muestran que las intervenciones educativas en el uso racional del medicamento en estudiantes de bachillerato han resultado efectivas tanto para mejorar el conocimiento de los estudiantes como para mejorar sus creencias respecto a la sobremedicalización y a la peligrosidad de los medicamentos. La efectividad de esta intervención es más notable en aquellos estudiantes con menor conocimiento o creencias menos deseables. No obstante, el tamaño del efecto varía en función de la variable de resultado.

El principal impacto ha tenido lugar sobre el conocimiento y la mejora media entre los participantes del grupo intervención fue de 1,4 puntos. Además, esa mejora destaca entre aquellos con un conocimiento inferior a la mediana en el momento previo a la intervención, con una mejora media que fue de 2,1 puntos en el grupo intervención.

El impacto de las intervenciones sobre las creencias relacionadas con los medicamentos ha sido más limitado, con diferencias medias entre grupos muy pequeñas, particularmente en el caso del nivel de creencia respecto a la peligrosidad de los medicamentos. Esto podría deberse a que el nivel medio inicial de la creencia en peligrosidad era inferior (más deseable) al de sobremedicalización (9,5 vs 12,0). 
En ambas subescalas de creencias (sobremedicalización y peligrosidad) los grupos de participantes que presentaron un nivel de creencias más negativo (superior a la mediana) en línea base se vieron más beneficiados de las intervenciones, ya que su nivel de creencias mejoró en mayor medida que en los que presentaron un nivel de creencia más positivo en línea base.

\section{Conclusiones}

Las intervenciones educativas sobre el uso de medicamentos en estudiantes de bachillerato se han demostrado efectivas y viables tanto para mejorar el conocimiento general sobre el proceso de uso del medicamento como para mejorar sus creencias y actitudes respecto a la sobremedicalización y peligrosidad de los medicamentos.

A su vez, se ha de destacar el alto grado de aceptación y satisfacción por parte del alumnado y personal docente con dichas intervenciones. Todo ello refuerza la convicción en la necesidad de implementar de forma definitiva esta actividad formativa como actividad educativa complementaria en el programa educativo de bachillerato impartida por farmacéuticos comunitarios.

\section{Agradecimientos}

A los Dres. María Rubio Valera, Joan Domènech Abella e Ignacio Aznar Lou, del Grupo de investigación PRISMA, del Parc Sanitari Sant Joan de Déu, Centro de Investigación Biomédica en Red de Epidemiología y Salud Pública (CIBERESP) como responsables del tratamiento estadístico, análisis de los resultados, y validación del cuestionario utilizado en este estudio.

A Antònia Ribas Moranta, licenciada en Psicopedagogía, miembro del Grupo para el Asesoramiento y la Innovación en Ámbitos Educativos para el Bienestar (GAEB), grupo asociado al Instituto de Investigación e Innovación Educativa de la Universitat de les Illes Balears. Gracias por su asesoramiento y orientación en el área pedagógica.

A Ana Oficialdegui López, farmacéutica, y responsable de Marketing Relacional en Laboratorios Cinfa, por su colaboración y fe inquebrantable en este proyecto.

A Laboratorios Cinfa, sin cuyo apoyo financiero no hubiera sido posible hacer este estudio.
A Pedro Molina, Alejandra Pop, Verónica Cuevas y Mario Vaillo, del staff de SEFAC, por su incondicional apoyo y labor logística en la puesta en marcha y desarrollo de este proyecto.

A los centros educativos y estudiantes participantes, por su gran acogida y aportación a este estudio.

A los coordinadores y 336 farmacéuticos voluntarios, sin los cuales este proyecto no se hubiera hecho realidad.

\section{Referencias bibliográficas}

1. World Health Organisation. Promoting rational use of medicines. [consultado 11 de noviembre de 2020]. Disponible en: https://www.who.int/activities/ promoting-rational-use-of-medicines/

2. Davis T, Wolf M, Bass P. Literacy and misunderstanding prescription drugs labels. Ann Intern Med. 2006;145:887-94. doi:10.7326/0003-4819-145-12-20061 2190-00144

3. Rigueira AI. Cumplimiento terapéutico: ¿qué conocemos de España? Aten Primaria 2001;27(8):559-68. doi:10.1016/ S0212-6567(01)78860-9

4. Richards M. Extent and causes of international variations in drug usage. A report for the Secretary of State for Health by Professor Sir Mike Richards CBE. London: Central Office of Information;2010. [consultado 11 de noviembre de 2020] Disponible en: https://assets. publishing.service.gov.uk/government/ uploads/system/uploads/attachment_ data/file/216249/dh_117977.pdf

5. García Delgado Pilar. Conocimiento del paciente sobres sus medicamentos. [Tesis doctoral. en internet]. Universidad de Granada 2008. [Consultado 7 julio 2016]; Disponible en: http://hera.ugr.es/ tesisugr/1771557x.pdf

6. Solá N, Cámara R, Cosín A, Dago A, Gutiérrez P, Salar L. D-valor programa de investigación y formación para farmacéuticos comunitarios informe de resultados. 2013. [consultado 31 Ago 2018]. Disponible en: https:// www.farmaceuticoscomunitarios.org/ es/journal-article/d-valor-valor-dispensacion-programa-investigacion-formacion-farmaceuticos

7. Campaña 2006-Uso racional del medicamento. Ministerio de Sanidad Servicios Sociales e Igualdad. España. [consultado 31 Ago 2016]. Disponible en: http://www. msc.es/campannas/campanas06/usomedicamento3.htm

8. Plaza Zamora, FJ. (2016). Alfabetización de medicamentos en farmacia comunitaria: preferencias, barreras y facilitadores de la información farmacoterapéutica. Proyecto de investigación. Disponible en: http://hdl.handle.net/10803/373635

9. Ley 29/2006, de 26 de julio, de garantías y uso racional de los medicamentos y productos sanitarios. «BOE» núm. 178, de 27 de julio de 2006. Disponible en: https://www.boe.es/buscar/act.php?id=BOE-A-2006-13554
10. Puig Soler R, Perramon Colet M, Zara Yahni C, Garcia Puig AM. Establecimiento de los conocimientos, actitudes y opiniones de la población sobre el uso racional de los medicamentos. Aten Primaria. 2015;47(7):446445. doi:10. 1016/j.aprim.2014.11.001

11. Herrera Carranza, J. Una reflexión sobre el valor humanístico intangible de la farmacia. Aula de la farmacia 2013; 9(99): 34-42.

12. Beléndez-Vázquez $M$, et al. Evaluación de las creencias sobre el tratamiento: validez y fiabilidad de la versión española del Beliefs about Medicines Questionnaire. Int J Clin Health Psychol, 2007; 7(3):767-779. Disponible en: http://aepc. es/ijchp/articulos_pdf/ijchp-251.pdf

13. Sapkota B, Rauniyar D, Shankar PR, Gupta GK, Thapa RK. Perception about Medicines among the General Public in the Semi-rural Areas of Nepal. Kathmandu Univ Med J (KUMJ). 2019 AprJun;17(66):93-100. PMID: 32632054. Disponible en: http://kumj.com.np/issue/66/93-100.pdf.

14. Klimaszova Z, Fazekas T, Kuzelova M. Adolescents' knowledge and perception of medicine risk. Pediatr Int. 2018 Aug;60(8):735-742. doi: 10.1111/ ped.13609. Epub 2018 Aug 3. PMID: 29856497. doi:10.1111/ped.13609

15. Klimaszova Z, Fazekas T, Kuzelova M. Relationship between adolescents' and their parents' attitudes toward medicines and awareness of the risk of medicines. Int J Clin Pharm. 2018 Dec;40(6):15011510. doi: 10.1007/s 11096-018-0723-8. Epub 2018 Aug 30. PMID: 30167969. doi:10.1007/s11096-018-0723-8

16. García-Delgado P, Gastelurrutia MA, Baena MI, Fisac F, Martínez F. Validación de un cuestionario para medir el conocimiento de los pacientes sobre sus medicamentos. Atención Primaria 2009; 41(12):661669. doi:10.1016/j.aprim.2009.03.011

17. Salmerón Rubio, Joaquín; García-Delgado, Pilar; Iglésias Ferreira, Paula; Mateus Santos, Henrique; Martínez-Martínez, Fernando. Validación del cuestionario de medida del conocimiento del paciente sobre su medicamento adaptado al portugués Ciência \&t Saúde Coletiva 2014; 19 (4):1141-1150. doi:10.1590/141381232014194.17612012

18. Huarte J, López J. Proyecto piloto sobre el impacto de la educación en el proceso del correcto uso del medicamento en estudiantes de bachiller. $1^{\text {a }}$ etapa ¿hay necesidad de educación?. Farmacéuticos Comunitarios. 2016 May 26; 8 (Supl 1). Disponible en: https://www.farmaceuticoscomunitarios. org/es/journal-article/proyecto-piloto-sobre-impacto-educacion-proceso-del-correcto-uso-del-medicamento

19. Huarte J, López J. Proyecto piloto sobre el impacto de la educación en el proceso del correcto uso del medicamento en estudiantes de bachiller. 2a etapa: valoración de la utilidad de la actividad educativa desarrollada. Farmacéuticos Comunitarios. 2016 May 26; 8(Supl1). Disponible en: https://www.farmaceuticoscomunitarios.org/es/journal-article/ proyecto-piloto-sobre-impacto-educacion-proceso-del-correcto-uso-del-medicamento- 0 